\title{
Monism, Pluralism and Relativism: New Essays on the Status of Logic
}

\author{
Daniel Cohnitz $\cdot$ Peter Pagin $\cdot$ Marcus Rossberg
}

Received: 5 March 2013/Accepted: 5 March 2013/Published online: 26 March 2013

(C) Springer Science+Business Media Dordrecht 2013

In logic, there are no morals. Everyone is at liberty to build his own logic, i.e. his own form of language as he wishes.

(Carnap, Logical Syntax of Language, 1934, \$17)

\section{What is the Status of Logic?}

The articles in this volume discuss the status of logic. What is the status of logic visà-vis our philosophical aspirations and projects? Is logic a neutral methodological framework within which we can develop and discuss our philosophical theories, or does the logic we chose as a framework already constrain the theories we try to develop in it? Of course, in order to be a useful framework, a logic should place some constraints on our theory-building and argumentation (by requiring, for example, logical validity of our arguments and perhaps logical consistency of our theories), but it should not set constraints on our philosophical theories that beg the question regarding the problem that we are trying to find an answer to, or so one might think.

D. Cohnitz

Department of Philosophy, University of Tartu, Ülikooli 18, 50090 Tartu, Estonia

e-mail: cohnitz@ut.ee

URL: http://daniel.cohnitz.de

P. Pagin

Department of Philosophy, Stockholm University, 10691 Stockholm, Sweden

e-mail: peter.pagin@philosophy.su.se

M. Rossberg $(\bowtie)$

Philosophy Department, University of Connecticut, Storrs, CT 06269-2054, USA

e-mail: marcus.rossberg@uconn.edu 
A further question about the status of logic is whether there is such a thing as the one correct logic to choose for our philosophical projects. This question perhaps arises regardless of whether logic can be, in the above sense, neutral with respect to every philosophical question, for different logics might place different constraints on our argumentation or on our theories. Is there a fact of the matter which logic is the correct one, or is the choice of logic merely a pragmatic matter, as Rudolf Carnap suggested in Carnap (1934)?

Whereas the first set of problems is about the question how logic is interwoven with our philosophical theories, the latter set turns on the discussion between monism, pluralism, and relativism about logic. In this introductory editorial we try to sketch the background of these discussions and explain how the articles in this collection contribute to them.

\section{What is Logic?}

Many think of logic as a particular sub-discipline of mathematics that studies a certain class of abstract, formal structures. The starting point for the study of philosophical logic, however, is not purely mathematical interest. It rather takes wing from the observation that certain (ordinary language) arguments share two interesting features: on the one hand, their premisses necessitate their conclusion, and on the other hand, they share a certain structure. Moreover, it seems that the first is the case because of the second. Presumably since Aristotle, the study of philosophical logic is the attempt to develop a formal theory that describes what these structures or patterns are and explains why some of these patterns have the interesting property of having only valid arguments as their instances.

\subsection{The Function of Logic}

Also since Aristotle, logic has been seen as a valuable methodological tool. After all, the fact that the logical validity of an argument seems to be a matter of instantiating a certain pattern naturally leads to the idea that logical validity is just a matter of form, and thus independent of the content or the subject matter of the argument. This would mean that logic has a topic-neutrality that would be immensely valuable in a discipline such as philosophy where everything is open for negotiation at any time. It provides the discipline with a methodological framework which does not presuppose any assumptions, for instance, regarding metaphysical questions. Sometimes (and in particular in the motivating introductory chapters of logic textbooks) it seems that this comprises the main role of logic in philosophy. Such a view somewhat overshadows the analytical role of logic as a theory of logical consequence mentioned above. ${ }^{1}$

\footnotetext{
1 It also overlooks the role of mathematical logic as a general framework for modeling philosophically interesting phenomena other than logical consequence. For a discussion of logic as a toolkit for modeling, see (Cohnitz 2012; Cook 2002; Shapiro 2006).
} 


\subsection{Logic as the Explication of Logical Consequence}

In what sense does logic provide a theory or explication of logical consequence? ${ }^{2}$ This may be best explained by distinguishing between logical validity as an actual property of ordinary language arguments or statements on the one hand, and their formal models on the other.

\subsubsection{The Explicandum}

Let us assume here that logical consequence is, in the first instance, a relation between statements of ordinary language. ${ }^{3}$ As we said above, one of the things that logic as a field of study tries to do, is to explain when and why arguments are valid. As also suggested above, this seems to have something to do with the argument form that particular arguments instantiate. The argument from 'Rudolf reads and Otto hums' to 'Otto hums' accordingly would appear to be valid because it instantiates a valid argument form: $\ulcorner\varphi$ and $\psi$; therefore $\psi\urcorner$. The validity of this argument form, in turn, appears largely to be down to the presence of the word 'and' in the premisein addition to the occurrence of the sentence $\psi$ both as one of the two sentences connected by 'and' in the premise and as the conclusion. If we replaced 'and' by 'unless', 'until', or 'only if', we would typically not be prepared to judge the argument to be valid. Let us call these words on which the validity of an argument forms hangs "logical words".

Ordinary language can be a messy affair, however. While we want to hold on to the thought that the schema above describes a valid argument form, ordinary language does hold felicitous counterexamples: we would not usually take 'You keep making a racket and you will not get any dessert' to entail either of its conjuncts. In fact, an utterance of this sentence would typically be designed to ensure that neither of the conjuncts - in particular, the first-turns out to be true, while the conjunction itself would be uttered in sincere belief of its truth. An obvious solution to the conundrum is to insist that, despite the presence of the word 'and', the sentence does not express a conjunction. The project of an explication of logical consequence has to accomplish two tasks: (i) it has to precisify the logical words by introducing technical terms in their place that behave in a more predictable way than their ordinary-language counterparts; ${ }^{4}$ (ii) it has to specify exactly what the logically valid argument forms are and what counts as an instance of such an argument form.

1935 was a great year for the explication of logical consequence: Gerhard Gentzen published his "Untersuchungen über das logische Schliessen" Gentzen (1935), and Alfred Tarski presented his account at the Congrés International de Philosophie Scientifique in Paris, "Über den Begriff der logischen Folgerung" Tarski (1936). The two major traditions in logic, the proof-theoretic and the model-theoretic approach, go back to these pivotal works by Gentzen and Tarski, respectively. It is worth noting,

\footnotetext{
${ }^{2}$ Regarding the notion of explication, compare Carnap (1950, §§2-4).

${ }^{3}$ For a discussion of this and alternative views, see Russell (2008).

4 Pace (Quine 2012).
} 
however, that a year before, Carnap (1934) had published his own proposal for an explication of logical consequence in his book Logische Syntax der Sprache (Logical Syntax of Language). Carnap's work has been superseded by Tarski's, of course. However, despite some disagreements, Carnap took Tarski's semantic approach largely to be a more elegant way of solving the problems he too was concerned with, and subsequently used it as a basis for his explorations into semantics. ${ }^{5}$ Carnap's early account includes the precise characterization of non-axiomatizable consequence relations for higher-order languages as well as for axiom systems that contain arithmetic. Carnap took all of his technical apparatus to be syntactic, rather than semantic, and his account of consequence no doubt has a decidedly syntactic feel. Many of the techniques and notions Carnap uses may be seen to fall into the remit of semantics, however, when viewed through Tarskian goggles. While Carnap's logical pluralism which is incorporated in Logical Syntax of Language is well known, a full appreciation of his explication of logical consequence is only just beginning. We here thus concentrate on Gentzen's and Tarski's accounts.

In both these accounts, the explication of the logical words is of particular importance (i.e., task (i) above). Borrowing the terminology of the model-theoretic approach, we will call these technical terms that serve as explicata of the logical words "logical constants" in discussion of either account. The familiar sign ' $\wedge$ ', for example, is used to designate the explicatum of the ordinary language word "and".

\subsubsection{The Proof-theoretic Conception}

The proof-theoretic approach famously aims to define the logical constants using socalled "operational rules". Following Gentzen, a logical constant is typically taken to be defined by its introduction rule, while its elimination rule is supposed to "follow from", in some sense, or be "in harmony" with, the meaning established by the introduction rule. ${ }^{6}$

Let us consider as an example the natural deduction formulation of the introduction and elimination rules for ' $\wedge$ ', i.e., the explication for the natural language "and".

$$
\frac{\varphi \psi \psi}{\varphi \wedge \psi} \wedge \mathrm{I} \quad \frac{\varphi \wedge \psi}{\varphi} \wedge \mathrm{E}_{1} \quad \frac{\varphi \wedge \psi}{\psi} \wedge \mathrm{E}_{2}
$$

The introduction rule, $\wedge \mathrm{I}$, defines what ' $\wedge$ ' is to mean. ' $\varphi$ ' and ' $\psi$ ' above the line stand in for (possibly logically complex) statements. $\wedge \mathrm{I}$ gives license to extend a proof that contains the lines $\ulcorner\varphi\urcorner$ and $\ulcorner\psi\urcorner$ by a line $\ulcorner\varphi \wedge \psi\urcorner$. In the case of ' $\wedge$ ', we have two elimination rules: $\wedge \mathrm{E}_{1}$ and $\wedge \mathrm{E}_{2}$ above.

The proof-theoretic proposal might be put thus:

(PT) An argument is valid if and only if there is a proof of its conclusion from its premisses that uses only the proper introduction- and elimination-rules of the logical constants.

\footnotetext{
5 See (Carnap 1942, x-xi, see also 1963, 60-64) and (Coffa 1987, 1991, ch. 16, esp. 301-302). This is in particular true of Tarski (1935), but also of Tarski (1936).

6 See Read (2000, 2010) for references and an insightful discussion.
} 
In fact, this formulation of the proposal might be too simplistic — at the very least, it requires qualification in order to accommodate the incompleteness results. Here is not the place, however, to discuss the various responses to the incompleteness results that proof-theorists have put forward.

\subsubsection{The Model-theoretic Conception}

The dominant model-theoretic tradition going back to Tarski starts from the idea that, in a valid argument, the conclusion is never false whenever the premisses are true. As is well known, varying re-interpretations of the sentences of a language are used in order to cash out this idea. Call an interpretation that makes a set of sentences true, a model of these sentences. The model-theoretic proposal, termed 'Tarski's Thesis' in Beall and Restall (2006), may thus be formulated thus (adapted from Tarski 1936):

(TT) An argument is valid if and only if every model of its premisses is also a model of its conclusion.

One can think of the re-interpretation of the sentences of the language as modeling all possible instances of an argument form. Any model of the premisses also being a model of the conclusion thus, in effect, comes down to this: no instance of the argument has true premisses and a false conclusion-just what we want from a valid argument.

One important point here is that the lexical items that designate the explicata of the logical words are not subject to re-interpretation. Their interpretation must remain constant (hence the term "logical constant")-for otherwise the reinterpretation would change the form of the argument, as suggested above-while the interpretation of all other lexical items varies across the models.

The proof- and model-theoretic techniques provide the tools for the explication of logical consequence, and many disparate explications have been proposed. Given the well-know plethora of logics_classical, intuitionistic, relevant, linear, fuzzy, quantum, connexive, ...-the question has often been: based on what criteria should we choose the correct logic? Our question however is: do we have to choose at all?

\section{One Logic or Many?}

With this background, we can begin to discuss what it would mean to be a monist, relativist or pluralist about logic. As we will see, there are several ways in which one might hold a version of pluralism about logic, consequently, there are different ways of being a monist about logic as well. Once these distinctions are in place, we can discuss which kind of pluralism would be philosophically exciting, and which would be trivial. 


\subsection{Monism, Pluralism, Relativism}

Stewart Shapiro (this volume) and Cook (2010) begin their discussion of the relationship between relativism and pluralism with a definition of relativism, based on the following general schema:

The correct account of $X$ is relative to $Y(\operatorname{Cook} 2010,493)$

One is a relativist about some phenomenon or topic $X$ if and only if one believes that the correct account of $X$ depends (is a function of) the $Y$-facts. Take, as an example, the case of etiquette. We normally believe that "the correct account of the conventional norms that constitute polite behavior ... seems to be a function of what culture one is living in" (Cook 2010, 493). Note that this account of relativism does not say much, unless it is further specified how the $X$ and the $Y$-facts are related. It is presumably true of almost any topic, that what should count as a correct account of that topic depends on some facts or other. ${ }^{7}$ But even if we require that the $Y$-facts are distinct from the $X$-facts, relativism is still different from pluralism about $X$.

Pluralism about some topic $X$ is the doctrine that there is more than one correct account of $X$. On the one hand, relativism about some topic $X$ might be true, without pluralism about $X$ being true. Just take a case in which the $X$-facts are a function of the $Y$-facts, but the $Y$-facts do not vary. In this case there is only one correct account of the $X$-facts. Of course, this would not be a very exciting case of relativism either, since the $Y$-facts are invariant, relativizing all claims about $X$ to the independent variable $Y$ would be redundant, but—as Cook points out—such a relativization might still make good sense. Just consider a possible world in which there is forever only a single culture. For that world, relativism about etiquette would still be correct and make sense, even though one should now be a monist about the correct account of the etiquette-facts.

The other direction too holds true: although pluralism does indeed often go hand in hand with relativism, there is a possibility for non-relativistic pluralism. We take it that the motivation for endorsing relativism sometimes departs from the attempt to combine pluralism about $X$ with truth-aptness of accounts of $X$. An expressivist about $X$-discourse can be a pluralist about $X$ without difficulty. ${ }^{8}$ If $\ulcorner\varphi$ is wrong $\urcorner$ is not truth-apt, then $\ulcorner\varphi$ is not wrong $\urcorner$ is not truth-apt either, and one can endorse pluralism without being forced into relativism. However, if one thinks that both claims are correct and truth-apt, the position is in danger of being inconsistent - and this inconsistency can then be resolved by endorsing relativism $(\ulcorner\varphi$ is wrong relative to $Y\urcorner$ and $\ulcorner\varphi$ is not wrong relative to $Z\urcorner$ ). In order to keep pluralism and relativism distinct, we will follow Cook (2010) in distinguishing between dependent pluralism and simple pluralism. A pluralist account of some topic $X$ is dependent, if

\footnotetext{
7 For example, to say that "the correct account of the conventional norms that constitute polite behavior ... seems to be a function of what culture one is living in", seems trivial precisely because we typically think that "the conventional norms that constitute polite behavior" are part of what counts as culture.

${ }^{8}$ Modulo all the other difficulties of expressivism.
} 
and only if it is the result of an underlying relativism. A pluralism that does not involve an underlying relativism is simple.

\subsection{Versions of Pluralism}

If one takes 'logic' to refer to formal logics, i.e. any pair $\langle L, \models\rangle$, where $L$ is a formal language and $\vDash$ is a consequence relation between sets of statements of $L$ on the one hand, and a single statement of $L$ on the other, then the claim that there is more than one logic is trivial, since there are infinitely many such formal systems. Cook argues that this pluralism, which he calls "mathematical logical pluralism" (MLP), isalthough trivial—nevertheless a necessary precondition for logical pluralism.

It seems clear that MLP is not what pluralists about logic have in mind. Neither is-what Cook calls-Philosophical Logic Pluralism (PLP), the claim that there is more than one logic that can fruitfully be applied to philosophically interesting phenomena (Cook 2010, 494), in particular, since there are several applications of logic in areas other than human reasoning. We get closer to interesting forms of logical pluralism if we restrict logic, as we did above, to the explication of logical consequence. But even in this case, logical pluralism can arise in several different ways. One way is language-relative logical pluralism, a form of dependent pluralism that arises once we realize that there are several formal languages that might serve as explications of logical consequence (which is the view Carnap defends in Carnap 1934 as his famous "principle of tolerance", one formulation of which we chose as a motto above).

If we stick to but one formal language, we can obtain a dependent logical pluralism in a different way, viz. by varying which part of the vocabulary of our language we consider to be logical constants (as, e.g., in Varzi 2002), or-as we shall see belowby varying which logical consequence relation is intended to be codified by formal means (Cook 2010, 500). Although this latter version too is a form of dependent pluralism (since the correct logic is still relative to the precisification of the pretheoretical logical consequence relation), this pluralism satisfies Cook's criterion for Substantial Logical Pluralism: there is more than one adequate explication of logical consequence even if we keep the formal language and the logical vocabulary fixed (Cook 2010, 496).

\section{The Contributions in This Volume}

Timothy Williamson's article 'Logic, Metalogic, and Neutrality' takes up the first set of questions we discussed in this introduction: the role of logic as a neutral arbiter for our metaphysical disputes. Williamson observes that the existing plurality of philosophical logics shows that logic is not, in that sense, neutral, since many of these philosophical logics are motivated precisely by metaphysical considerations. Metalogic, the framework in which we discuss the differences between those alternative logics, is perhaps the best candidate for a set of logical principles that are accepted widely enough to play the role of neutral arbiter, but Williamson also frustrates that hope. Metalogic turns out not to be metaphysically 
neutral either. But, Williamson argues, we should learn to live with this result. Logic just is not metaphysically neutral, and it does not have to be in order to play its role as a scientific theory.

In 'Should Anti-Realists be Anti-Realists about Anti-Realism?' Roy T. Cook addresses a similar question. He discusses whether the arguments that proponents of "Dummettian" anti-realism provide are strong enough to constrain the choice of logics for a Dummettian anti-realist to intuitionistic logic, or "merely" require a revision of the law of excluded middle, but otherwise leave a certain range of logics open to be anti-realistically acceptable. Cook argues that indeed the anti-realist arguments only support the weaker claim. This leaves it open to the anti-realist to be a pluralist about logic, provided the anti-realist is careful about what logics she includes in this pluralism, and provided she is careful in how she formulates her pluralism. In that sense, the anti-realist should be anti-realist about anti-realism.

Nikolaj Jang Lee Linding Pedersen looks at the interrelations between metaphysical pluralism (according to which there are several ways of being), alethic pluralism (according to which there are several ways of being true), and logical pluralism (according to which there are several ways of being valid) in his 'Pluralism $\times 3$ : truth, logic, metaphysics'. Pedersen argues that (some version of) alethic pluralism indeed supports logical pluralism and that both are partly founded on metaphysical pluralism.

Whereas the first three papers in this volume investigate from different perspectives how metaphysical and epistemological views set constraints on logic and logical pluralism, the remaining papers in this volume focus more directly on the idea of logical pluralism as such.

In 'Pluralism and Proof' Greg Restall explicates logical pluralism in terms of different standards of proof, as an alternative to the model-theoretic approach taken in Beall and Restall (2006). In their book, Beall and Restall argued for a substantial logical pluralism in Cook's sense (explained above in Sect. 3.2), viz. that the explication of logical consequence provided by the Generalized Tarski Thesis:

(GTT) An argument is valid ${ }_{X}$ if and only if, in every case ${ }_{X}$ in which the premisses are true, the conclusion is true

allows for different consequence relations, relative to the notion of 'case ${ }_{X}$ ' that is plugged into the scheme. Now Restall discusses how different restrictions on classical derivations yield derivations for intuitionistic logic and dual-intuitionistic logic. In the final part of the paper Restall explores how this relates to, and further motivates, the pluralism developed in Beall and Restall (2006).

Manuel Bremer's 'Restall and Beall on Logical Pluralism-A Critique' also engages with the account developed in Beall and Restall (2006). However, like Williamson, Bremer is much more skeptical about the feasibility of the pluralist's position. Bremer formulates seven challenges to logical pluralism, some of which question the precise nature of logical pluralism as a doctrine (is it an empirical claim or arrived at a priori? how can the idea of logical pluralism be reconciled with claims about the determinateness of logical form made by linguists?), some of which see Beall and Restall's argumentation in tension with the idea of logical pluralism (what is the status of the logic that Beall and Restall adopt in arguing for 
logical pluralism? what is the status of the metatheory in which the alternative logics are presented?).

Jc Beall, on the other hand, tries to shed some light on how logic can serve as a normative guide to reasoning despite logical pluralism in his 'Strict-choice validities: a note on a familiar pluralism'. Beall shows that multiple conclusion $\mathrm{LP}^{+}$may leave us with a set of consequences, informing us that these are valid, but not guiding us in what inferences we should draw. If it requires a further logic to guide us in our inferences, then this gives rise to a further form of pluralism.

Stewart Shapiro shifts the focus from logical pluralism to logical relativism in 'Structures and logics: a case for (a) relativism'. Shapiro adopts the notion of relativism we discussed above, Sect. 3.1, according to which Beall and Restall's "pluralism" is also a form of relativism, since the logics considered by Beall and Restall are relative to the notion of 'case ${ }_{X}$ ' in (GTT). Shapiro then goes on to defend a relativism about logical consequence, where logical consequence is relative to structures. Shapiro shows that certain coherent mathematical structures constrain the applicability of logical terminology. For example, classical negation cannot be added to smooth infinitesimal analysis without rendering the theory inconsistent. This is a form of relativism of logic to structures that does not deflate all disputes about what should be the logic proper for a given structure.

Graham Priest's analysis in 'Logical Pluralism: Another Application for Chunk and Permeate' deals with a related case. If logical pluralism is motivated by some form of relativism, such that logic, or logical consequence, is relative to the kind of object one is reasoning about (e.g., mathematical constructions, quantum objects, or medium-sized dry goods) an interesting problem arises: what to do in cases where the reasoning concerns a mix of kinds of objects? Which logic applies to these mixed cases? Simply taking the intersection of all logics applicable to pure kinds will leave us with a very weak logic; developing a sui generis logic for each individual mix also seems unsatisfactory, since the objects in the mix already have "their" logic. Priest suggests a model to deal with such cases, which partitions our reasoning in discrete "chunks" and allows information to "permeate" between those chunks, while the logic from one chunk to the next may vary.

Acknowledgments It was the aim of the conference "Logical Pluralism", which took place in Tartu, Estonia, August 27th-31st, 2008, to bring together philosophers of logic to discuss the status of logic and the feasibility of logical pluralism in particular. Some of the papers in this issue were presented at this conference, others contributed by colleagues who could not attend the conference at Tartu. We would like to thank the Bank of Sweden Tercentenary Fund, and the Department of Philosophy at the University of Tartu for financial and institutional support, and acknowledge the support of the Estonian Science Foundation grants SF0180110s08 and SFLFI11085E. We also thank the authors and referees of the articles for their work, and Hannes Leitgeb for his support of this project.

\section{References}

Beall, J. C., \& Restall, G. (2006). Logical pluralism. Oxford: Oxford University Press. Carnap, R. (1934). Logische Syntax der Sprache. Wien: Springer.

Carnap, R. (1942). Introduction to semantics. Cambridge, MA: Harvard University Press.

Carnap, R. (1950). Logical foundations of probability. Chicago: Chicago University Press. 
Carnap, R. (1963). Intellectual autobiography. In P. A. Schilpp (Ed.), The philosophy of Rudolf Carnap, volume XI of library of living philosophers (pp. 1-84). Chicago: Chicago University Press.

Coffa, A. (1987). Carnap, Tarski and the search for truth. Noûs, 21(4), 547-572.

Coffa, A. (1991). In L. Wessels (Ed.), The semantic tradition from Kant to Carnap: to the Vienna station. Cambridge: Cambridge University Press.

Cohnitz, D. (2012). The logic(s) of modal knowledge. In R. Greg, \& R. Gillian (Eds.), New waves in philosophical logic. Basingstoke: Palgrave Macmillan.

Cook, R. T. (2002). Vagueness and mathematical precision. Mind, 111, 225-247.

Cook, R. T. (2010). Let a thousand flowers bloom: A tour of logical pluralism. Philosophy Compass, 5(6), 492-504.

Gentzen, G. (1935). Untersuchungen über das logische Schliessen. Mathematische Zeitschrift, 39(2-3), 176-210, 405-431.

Quine, W. V. (2012). Truth by convention. In The ways of paradox (pp. 77-106).

Quine, W. V. (1976). The Ways of Paradox and other essays, 2nd, revised and enlarged edition. Cambridge, MA: Harvard University Press.

Read, S. (2000). Harmony and autonomy in classical logic. Journal of Philosophical Logic, 29(2), 123-154.

Read, S. (2010). General-elimination harmony and the meaning of the logical constants. Journal of Philosophical Logic, 39(5), 123-154.

Russell, G. (2008). One true logic? Journal of Philosophical Logic, 67(6), 593-611.

Shapiro, S. (2006). Vagueness in context. Oxford: Oxford University Press.

Tarski, A. (1935). Der Wahrheitsbegriff in den formalisierten Sprachen. Studia Philosophica, 1, $261-405$.

Tarski, A. (1936). Über den Begriff der logischen Folgerung. Actes du Congrès International de Philosophie Scientifique, 7, 1-11.

Varzi, A. (2002). On logical relativity. Philosophical Issues, 10, 197-219. 